\title{
COMPLETE MONOTONICITY OF A DIFFERENCE DEFINED BY FOUR DERIVATIVES OF A FUNCTION CONTAINING TRIGAMMA FUNCTION
}

\author{
FENG QI \\ Dedicated to people facing and battling COVID-19
}

\begin{abstract}
In the paper, by virtue of convolution theorem for the Laplace transforms, logarithmic convexity of the gamma function, Bernstein's theorem for completely monotonic functions, and other techniques, the author finds necessary and sufficient conditions for a difference defined by four derivatives of a function containing trigamma function to be completely monotonic. Moreover, by virtue of Čebyšev integral inequality, the author presents logarithmic convexity of the sequence of polygamma functions.
\end{abstract}

\section{Contents}

1. Preliminaries and motivations 1

2. Lemmas 4

3. Necessary and sufficient conditions of complete monotonicity 5

\begin{tabular}{|lll}
\hline 4. & Logarithmic convexity of the sequence of polygamma functions & 8
\end{tabular}

5. Remarks 9

References 9

\section{Preliminaries and motivations}

In the literature [1, Section 6.4], the function

$$
\Gamma(z)=\int_{0}^{\infty} t^{z-1} e^{-t} \mathrm{~d} t, \quad \Re(z)>0
$$

and its logarithmic derivative

$$
\psi(z)=[\ln \Gamma(z)]^{\prime}=\frac{\Gamma^{\prime}(z)}{\Gamma(z)}
$$

are called Euler's gamma function and digamma function respectively. Further, the functions $\psi^{\prime}(z), \psi^{\prime \prime}(z), \psi^{\prime \prime \prime}(z)$, and $\psi^{(4)}(z)$ are known as trigamma, tetragamma, pentagamma, and hexagamma functions respectively. All the derivatives $\psi^{(k)}(z)$

2010 Mathematics Subject Classification. Primary 33B15; Secondary 26A48, 26D07, 33B10, 44A10, 44A35.

Key words and phrases. necessary and sufficient condition; complete monotonicity; logarithmic convexity; gamma function; polygamma function; convolution theorem for the Laplace transforms; Bernstein's theorem for completely monotonic function; difference; derivative; trigamma function; majorization; Schur-convexity.

This paper was typeset using $\mathcal{A} \mathcal{M} \mathcal{S}$ - $\mathrm{LAT}_{\mathrm{E}} \mathrm{X}$. 
for $k \in\{0\} \cup \mathbb{N}$ are known as polygamma functions. There have been a number of literature dedicated to studying of the gamma and polygamma functions. See the papers [2, 3, 4, 7, 8, 13, 15, 20, 21, 22, 24, 26, 32, 33, and closely related references therein.

Recall from Chapter XIII in 11, Chapter 1 in 27, and Chapter IV in 30, that, if a function $f(x)$ on an interval $I$ has derivatives of all orders on $I$ and satisfies $(-1)^{n} f^{(n)}(x) \geq 0$ for $x \in I$ and $n \in\{0\} \cup \mathbb{N}$, where $\mathbb{N}$ denotes the set of all positive integers, then we call $f(x)$ a completely monotonic function on $I$. There have been a number of literature dedicated to studying of completely monotonic functions and their degrees. See the papers [2, 3, 4, 5, 7, 8, 13, 15, 20, 21, 22, 26, 32, 33. and closely related references therein.

Let $\alpha=\left(\alpha_{1}, \alpha_{2}, \ldots, \alpha_{n}\right)$ and $\beta=\left(\beta_{1}, \beta_{2}, \ldots, \beta_{n}\right) \in \mathbb{R}^{n}$. A $n$-tuple $\alpha$ is said to strictly majorize $\beta$ (in symbols $\alpha \succ \beta)$ if $\left(\alpha_{[1]}, \alpha_{[2]}, \ldots, \alpha_{[n]}\right) \neq\left(\beta_{[1]}, \beta_{[2]}, \ldots, \beta_{[n]}\right)$, $\sum_{i=1}^{k} \alpha_{[i]} \geq \sum_{i=1}^{k} \beta_{[i]}$ for $1 \leq k \leq n-1$, and $\sum_{i=1}^{n} \alpha_{i}=\sum_{i=1}^{n} \beta_{i}$, where $\alpha_{[1]} \geq$ $\alpha_{[2]} \geq \cdots \geq \alpha_{[n]}$ and $\beta_{[1]} \geq \beta_{[2]} \geq \cdots \geq \beta_{[n]}$ are rearrangements of $\alpha$ and $\beta$ in a descending order. A real-valued function $\phi$ defined on a set $\mathscr{A} \subset \mathbb{R}^{n}$ is said to be Schur-convex on $\mathscr{A}$ if $\boldsymbol{x} \prec \boldsymbol{y}$ for $\boldsymbol{x}, \boldsymbol{y} \in \mathscr{A}$ means $\phi(\boldsymbol{x})<\phi(\boldsymbol{y})$. See [9, p. 8, Definition A.1] [9, p. 80, Definition A.1]. There have been a lot of literature such as the papers [6, 26, 28, 31, 34, 35] dedicated to investigation of Schur-convexity.

Let $\Phi(x)=x \psi^{\prime}(x)-1=x\left[\psi^{\prime}(x)-\frac{1}{x}\right]$ on $(0, \infty)$. In [17, Theorem 4.1] and [20, Theorem 4], the author gave the following necessary and sufficient conditions and double inequality:

(1) if and only if $\alpha \geq 2$, the function $\mathfrak{H}_{\alpha}(x)=\Phi^{\prime}(x)+\alpha \Phi^{2}(x)$ is completely monotonic on $(0, \infty)$;

(2) if and only if $\alpha \leq 1$, the function $-\mathfrak{H}_{\alpha}(x)$ is completely monotonic on $(0, \infty)$

(3) the double inequality $-2<\frac{\Phi^{\prime}(x)}{\Phi^{2}(x)}<-1$ is valid and sharp in the sense that the lower and upper bounds -2 and -1 cannot be replaced by any bigger and smaller ones respectively.

In 17, Theorem 1.1], the author found the following necessary and sufficient conditions and limits:

(1) if and only if $\beta \geq 2$, the function $H_{\beta}(x)=\frac{\Phi^{\prime}(x)}{\Phi^{\beta}(x)}$ is decreasing on $(0, \infty)$, with the limits

$$
\lim _{x \rightarrow 0^{+}} H_{\beta}(x)=\left\{\begin{array}{ll}
-1, & \beta=2 \\
0, & \beta>2
\end{array} \text { and } \lim _{x \rightarrow \infty} H_{\beta}(x)= \begin{cases}-2, & \beta=2 \\
-\infty, & \beta>2 ;\end{cases}\right.
$$

(2) if $\beta \leq 1$, the function $H_{\beta}(x)$ is increasing on $(0, \infty)$, with the limits

$$
H_{\beta}(x) \rightarrow \begin{cases}-\infty, & x \rightarrow 0^{+} \\ 0, & x \rightarrow \infty\end{cases}
$$

For $k \in\{0\} \cup \mathbb{N}$ and $\lambda_{k}, \mu_{k} \in \mathbb{R}$, let

$$
\mathfrak{J}_{k, \lambda_{k}}(x)=\Phi^{(2 k+1)}(x)+\lambda_{k}\left[\Phi^{(k)}(x)\right]^{2} \quad \text { and } \quad J_{k, \mu_{k}}(x)=\frac{\Phi^{(2 k+1)}(x)}{\left[(-1)^{k} \Phi^{(k)}(x)\right]^{\mu_{k}}}
$$

on $(0, \infty)$. In [12, Theorems 3.1 and 4.1], the author gave the following necessary and sufficient conditions, limits, and double inequality: 
(1) if and only if $\lambda_{k} \geq \frac{(2 k+2) !}{k !(k+1) !}$, the function $\mathfrak{J}_{k, \lambda_{k}}(x)$ is completely monotonic on $(0, \infty)$;

(2) if and only if $\lambda_{k} \leq \frac{1}{2} \frac{(2 k+2) !}{k !(k+1) !}$, the function $-\mathfrak{J}_{k, \lambda_{k}}(x)$ is completely monotonic on $(0, \infty)$;

(3) if and only if $\mu_{k} \geq 2$, the function $J_{k, \mu_{k}}(x)$ is decreasing on $(0, \infty)$, with the limits

$$
\lim _{x \rightarrow 0^{+}} J_{k, \mu_{k}}(x)= \begin{cases}-\frac{1}{2} \frac{(2 k+2) !}{k !(k+1) !}, & \mu_{k}=2 \\ 0, & \mu_{k}>2\end{cases}
$$

and

$$
\lim _{x \rightarrow \infty} J_{k, \mu_{k}}(x)= \begin{cases}-\frac{(2 k+2) !}{k !(k+1) !}, & \mu_{k}=2 \\ -\infty, & \mu_{k}>2 ;\end{cases}
$$

(4) if $\mu_{k} \leq 1$, the function $J_{k, \mu_{k}}(x)$ is increasing on $(0, \infty)$, with the limits

$$
J_{k, \mu_{k}}(x) \rightarrow \begin{cases}-\infty, & x \rightarrow 0^{+} \\ 0, & x \rightarrow \infty\end{cases}
$$

(5) the double inequality

$$
-\frac{(2 k+2) !}{k !(k+1) !}<\frac{\Phi^{(2 k+1)}(x)}{\left[\Phi^{(k)}(x)\right]^{2}}<-\frac{1}{2} \frac{(2 k+2) !}{k !(k+1) !}
$$

is valid on $(0, \infty)$ and sharp in the sense that the lower and upper bounds cannot be replaced by any larger and smaller numbers respectively.

For $m, n \in\{0\} \cup \mathbb{N}$ and $\omega_{m, n} \in \mathbb{R}$, let

$$
Y_{m, n}(x)=\frac{\Phi^{(m+n+1)}(x)}{\Phi^{(m)}(x) \Phi^{(n)}(x)}
$$

and

$$
\mathcal{Y}_{m, n ; \omega_{m, n}}(x)=\Phi^{(m+n+1)}(x)+\omega_{m, n} \Phi^{(m)}(x) \Phi^{(n)}(x) .
$$

In [23, Theorems 3.1 and 4.1], the following necessary and sufficient conditions, monotonicity, complete monotonicity, and double inequality were established:

(1) the function $Y_{m, n}(x)$ is decreasing in $x$ from $(0, \infty)$ onto the open interval

$$
\left(-\frac{2(m+n+1) !}{m ! n !},-\frac{(m+n+1) !}{m ! n !}\right)
$$

(2) if and only if $\omega_{m, n} \leq \frac{(m+n+1) !}{m ! n !}$, the function $(-1)^{m+n+1} \mathcal{Y}_{m, n ; \omega_{m, n}}(x)$ is completely monotonic on $(0, \infty)$;

(3) if and only if $\omega_{m, n} \geq \frac{2(m+n+1) !}{m ! n !}$, the function $(-1)^{m+n} \mathcal{Y}_{m, n ; \omega_{m, n}}(x)$ is completely monotonic on $(0, \infty)$;

(4) the double inequality

$$
-\frac{2(m+n+1) !}{m ! n !}<Y_{m, n}(x)<-\frac{(m+n+1) !}{m ! n !}
$$

is valid on $(0, \infty)$ and sharp in the sense that the lower and upper bounds cannot be replaced by any larger and smaller numbers respectively. 
In this paper, we would like to consider monotonicity of the function

$$
\boldsymbol{Y}_{i, j ; \ell, m}(x)=\frac{\Phi^{(i)}(x) \Phi^{(j)}(x)}{\Phi^{(\ell)}(x) \Phi^{(m)}(x)}
$$

and complete monotonicity of the function

$$
\mathfrak{Y}_{i, j ; \ell, m ; \Omega_{i, j ; \ell, m}}(x)=(-1)^{i+j} \Phi^{(i)}(x) \Phi^{(j)}(x)-(-1)^{\ell+m} \Omega_{i, j ; \ell, m} \Phi^{(\ell)}(x) \Phi^{(m)}(x) \quad(1.1)
$$

on $(0, \infty)$, where $i, j, \ell, m \geq 0$ are integers such that $(i, j) \succ(\ell, m)$. Figure 1 plotted by the software Mathematica hints that the function $\boldsymbol{Y}_{17,11 ; 15,13}(x)$ is not monotonic in $x \in(0, \infty)$. Therefore, in this paper, we will only consider the

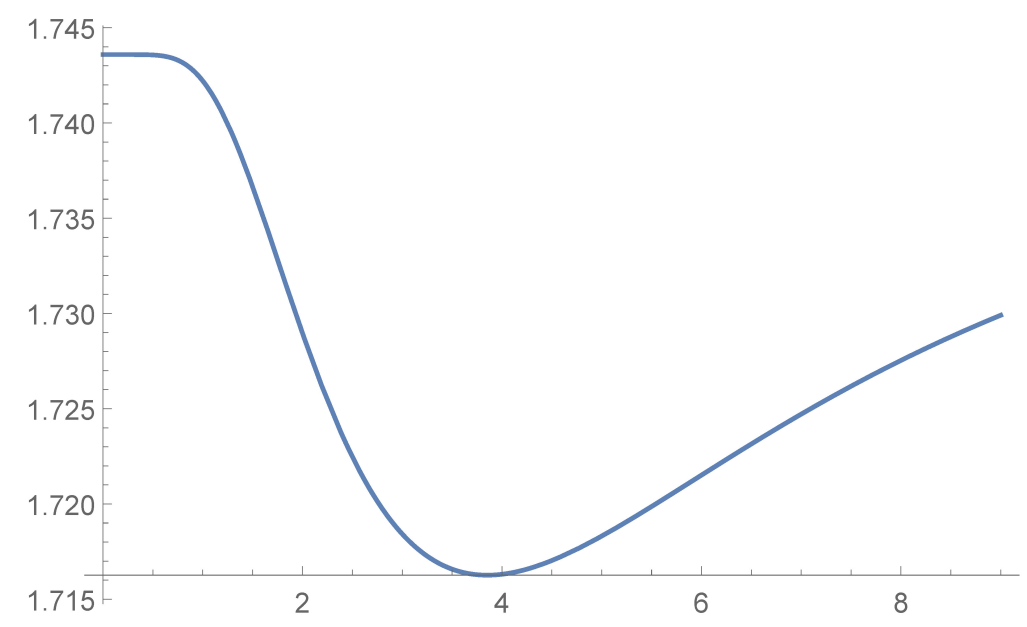

Figure 1. The graph of the function $\boldsymbol{Y}_{17,11 ; 15,13}(x)$ on $(0,9)$

functions $\pm \mathfrak{Y}_{i, j ; \ell, m ; \Omega_{i, j ; \ell, m}}(x)$ and find necessary and sufficient conditions on $\Omega_{i, j ; \ell, m}$ for $\pm \mathfrak{Y}_{i, j ; \ell, m ; \Omega_{i, j ; \ell, m}}(x)$ to be completely monotonic on $(0, \infty)$.

Moreover, we will also present that the sequence of polygamma functions $\psi^{(n)}(x)$ for $n \in \mathbb{N}$ is logarithmic convex.

\section{LEMMAS}

The following lemmas are necessary in this paper.

Lemma 2.1 ([17, Lemma 2.3]). Let

$$
h(t)= \begin{cases}\frac{e^{t}\left(e^{t}-1-t\right)}{\left(e^{t}-1\right)^{2}}, & t \neq 0 \\ \frac{1}{2}, & t=0 .\end{cases}
$$

For any fixed $t>0$, the function $h(s t) h((1-s) t)$ is increasing in $s \in\left(0, \frac{1}{2}\right)$.

Lemma 2.2 (Convolution theorem for the Laplace transforms [30, pp. 91-92]). Let $f_{k}(t)$ for $k=1,2$ be piecewise continuous in arbitrary finite intervals included in $(0, \infty)$. If there exist some constants $M_{k}>0$ and $c_{k} \geq 0$ such that $\left|f_{k}(t)\right| \leq M_{k} e^{c_{k} t}$ for $k=1,2$, then

$$
\int_{0}^{\infty}\left[\int_{0}^{t} f_{1}(u) f_{2}(t-u) \mathrm{d} u\right] e^{-s t} \mathrm{~d} t=\int_{0}^{\infty} f_{1}(u) e^{-s u} \mathrm{~d} u \int_{0}^{\infty} f_{2}(v) e^{-s v} \mathrm{~d} v .
$$


Lemma 2.3 ([14, Lemma 2.6]). For $m, n, p, q \in \mathbb{N}$ such that $(p, q) \succ(m, n)$, the function

$$
\frac{s^{m-1}(1-s)^{n-1}+(1-s)^{m-1} s^{n-1}}{s^{p-1}(1-s)^{q-1}+(1-s)^{p-1} s^{q-1}}
$$

is increasing in $s$ from $\left(0, \frac{1}{2}\right)$ onto $(0,1)$.

Lemma 2.4. For $i, j, \ell, m \in\{0\} \cup \mathbb{N}$ with $(i, j) \succ(\ell, m)$, the inequality $i ! j !>\ell ! m !$ is valid.

Proof. It is well-knon that the function $\Gamma(x)$ is logarithmically convex on $(0, \infty)$. See [18, Corollary 1] and [29, p. 42, Section 3.1.2]. In [9, p. 92, C.1.a (i)], it is stated that, if $g(x)$ is convex on an interval $I$, then the function $\phi(\boldsymbol{x})=\sum_{k=1}^{n} g\left(x_{k}\right)$ is Schur-convex on $I^{n}$, where $\boldsymbol{x}=\left(x_{1}, x_{2}, \ldots, x_{n}\right) \in I^{n}$. Hence, taking $g(x)=\ln \Gamma(x)$, $n=2$, and $I=(0, \infty)$ yields that, when $(i, j) \succ(\ell, m)$ and $i, j, \ell, m \in \mathbb{N}$, we have

$$
\ln \Gamma(i)+\ln \Gamma(j)>\ln \Gamma(\ell)+\ln \Gamma(m),
$$

that is,

$$
(i-1) !(j-1) !=\Gamma(i) \Gamma(j)>\Gamma(\ell) \Gamma(m)=(\ell-1) !(m-1) ! .
$$

The proof of Lemma 2.4 is complete.

Lemma 2.5 (Bernstein's theorem [30, p. 161, Theorem 12b]). A function $f(x)$ is completely monotonic on $(0, \infty)$ if and only if

$$
f(x)=\int_{0}^{\infty} e^{-x t} \mathrm{~d} \sigma(t), \quad x \in(0, \infty),
$$

where $\sigma(s)$ is non-decreasing and the integral in 2.1) converges for $x \in(0, \infty)$.

\section{NeCESSARY AND SUfFicient CONDITIONS OF COMPLETE MONOTONICITY}

In this section, we find necessary and sufficient conditions on $\Omega_{i, j, \ell, m}$ such that the functions $\pm \mathfrak{Y}_{i, j ; \ell, m ; \Omega_{i, j ; \ell, m}}(x)$ defined by (1.1) are completely monotonic.

Theorem 3.1. For $i, j, \ell, m \geq 0$ are integers such that $(i, j) \succ(\ell, m)$,

(1) if $\Omega_{i, j ; \ell, m} \leq 1$, the function $\mathfrak{Y}_{i, j ; \ell, m ; \Omega_{i, j ; \ell, m}}(x)$ defined in 1.1] is completely monotonic on $(0, \infty)$;

(2) if and only if $\Omega_{i, j ; \ell, m} \geq \frac{i ! j !}{\ell ! m !}$, the function $-\mathfrak{Y}_{i, j ; \ell, m ; \Omega_{i, j, \ell, m}}(x)$ is completely monotonic on $(0, \infty)$;

(3) the double inequality

$$
1<\frac{\Phi^{(i)}(x) \Phi^{(j)}(x)}{\Phi^{(\ell)}(x) \Phi^{(m)}(x)}<\frac{i ! j !}{\ell ! m !}
$$

is valid on $(0$, infty) and the right hand side inequality is sharp in the sense that the number $\frac{i ! j !}{\ell ! m !}$ can not be replaced by any smaller one.

Proof. In the proof of [20, Theorem 4], the author derived an integral representation

$$
\Phi(x)=\int_{0}^{\infty} h(t) e^{-x t} \mathrm{~d} t,
$$

where $h(t)$ is defined in Lemma 2.1. By virtue of Lemma 2.2, we acquire

$$
\mathfrak{Y}_{i, j ; \ell, m ; \Omega_{i, j ; \ell, m}}(x)=\int_{0}^{\infty} t^{i} h(t) e^{-x t} \mathrm{~d} t \int_{0}^{\infty} t^{j} h(t) e^{-x t} \mathrm{~d} t
$$




$$
\begin{aligned}
& -\Omega_{i, j ; \ell, m} \int_{0}^{\infty} t^{\ell} h(t) e^{-x t} \mathrm{~d} t \int_{0}^{\infty} t^{m} h(t) e^{-x t} \mathrm{~d} t \\
= & \int_{0}^{\infty}\left[\int_{0}^{t} u^{i}(t-u)^{j} h(u) h(t-u) \mathrm{d} u\right] e^{-x t} \mathrm{~d} t \\
& -\Omega_{i, j ; \ell, m} \int_{0}^{\infty}\left[\int_{0}^{t} u^{\ell}(t-u)^{m} h(u) h(t-u) \mathrm{d} u\right] e^{-x t} \mathrm{~d} t \\
= & \int_{0}^{\infty}\left[\frac{\int_{0}^{t} u^{i}(t-u)^{j} h(u) h(t-u) \mathrm{d} u}{\int_{0}^{t} u^{\ell}(t-u)^{m} h(u) h(t-u) \mathrm{d} u}-\Omega_{i, j ; \ell, m}\right] \\
& \times\left[\int_{0}^{t} u^{\ell}(t-u)^{m} h(u) h(t-u) \mathrm{d} u\right] e^{-x t} \mathrm{~d} t \\
= & \int_{0}^{\infty}\left[\frac{\int_{0}^{1} s^{i}(1-s)^{j} h(s t) h((1-s) t) \mathrm{d} s}{\int_{0}^{1} s^{\ell}(1-s)^{m} h(s t) h((1-s) t) \mathrm{d} s}-\Omega_{i, j ; \ell, m}\right] \\
& \times\left[\int_{0}^{t} u^{\ell}(t-u)^{m} h(u) h(t-u) \mathrm{d} u\right] e^{-x t} \mathrm{~d} t \\
= & \int_{0}^{\infty}\left[\frac{\int_{0}^{1 / 2}\left[s^{i}(1-s)^{j}+s^{j}(1-s)^{i}\right] h(s t) h((1-s) t) \mathrm{d} s}{\int_{0}^{1 / 2}\left[s^{\ell}(1-s)^{m}+s^{m}(1-s)^{\ell}\right] h(s t) h((1-s) t) \mathrm{d} s}\right. \\
& \left.-\Omega_{i, j ; \ell, m}\right]\left[\int_{0}^{t} u^{\ell}(t-u)^{m} h(u) h(t-u) \mathrm{d} u\right] e^{-x t} \mathrm{~d} t .
\end{aligned}
$$

By Lemma 2.3. we obtain that the double inequality

$$
1<\frac{s^{i}(1-s)^{j}+(1-s)^{j} s^{i}}{s^{\ell}(1-s)^{m}+(1-s)^{m} s^{\ell}}<\infty
$$

is valid and sharp for $s \in\left(0, \frac{1}{2}\right)$ and $(i, j) \succ(\ell, m)$. This means that

$$
\frac{\int_{0}^{1 / 2}\left[s^{i}(1-s)^{j}+s^{j}(1-s)^{i}\right] h(s t) h((1-s) t) \mathrm{d} s}{\int_{0}^{1 / 2}\left[s^{\ell}(1-s)^{m}+s^{m}(1-s)^{\ell}\right] h(s t) h((1-s) t) \mathrm{d} s}>1
$$

for $t \in(0, \infty)$ and $(i, j) \succ(\ell, m)$. Therefore, when $\Omega_{i, j ; \ell, m} \leq 1$ and $(i, j) \succ(\ell, m)$, the function $\mathfrak{Y}_{i, j ; \ell, m ; \Omega_{i, j ; \ell, m}}(x)$ is completely monotonic on $(0, \infty)$.

It is easy to see that

$$
\begin{aligned}
& \lim _{t \rightarrow 0^{+}} \frac{\int_{0}^{1 / 2}\left[s^{i}(1-s)^{j}+s^{j}(1-s)^{i}\right] h(s t) h((1-s) t) \mathrm{d} s}{\int_{0}^{1 / 2}\left[s^{\ell}(1-s)^{m}+s^{m}(1-s)^{\ell}\right] h(s t) h((1-s) t) \mathrm{d} s} \\
= & \lim _{t \rightarrow \infty} \frac{\int_{0}^{1 / 2}\left[s^{i}(1-s)^{j}+s^{j}(1-s)^{i}\right] h(s t) h((1-s) t) \mathrm{d} s}{\int_{0}^{1 / 2}\left[s^{\ell}(1-s)^{m}+s^{m}(1-s)^{\ell}\right] h(s t) h((1-s) t) \mathrm{d} s} \\
= & \frac{\int_{0}^{1 / 2}\left[s^{i}(1-s)^{j}+s^{j}(1-s)^{i}\right] \mathrm{d} s}{\int_{0}^{1 / 2}\left[s^{\ell}(1-s)^{m}+s^{m}(1-s)^{\ell}\right] \mathrm{d} s}=\frac{B(i+1, j+1)}{B(\ell+1, m+1)}=\frac{i ! j !}{\ell ! m !} .
\end{aligned}
$$

Let

$$
S_{i, j ; \ell, m}(t)=\int_{0}^{1 / 2}\left[s^{i}(1-s)^{j}+s^{j}(1-s)^{i}\right] h(s t) h((1-s) t) \mathrm{d} s
$$




$$
\begin{aligned}
& -\frac{i ! j !}{\ell ! m !} \int_{0}^{1 / 2}\left[s^{\ell}(1-s)^{m}+s^{m}(1-s)^{\ell}\right] h(s t) h((1-s) t) \mathrm{d} s \\
= & \int_{0}^{1 / 2}\left(\left[s^{i}(1-s)^{j}+s^{j}(1-s)^{i}\right]\right. \\
& \left.-\frac{i ! j !}{\ell ! m !}\left[s^{\ell}(1-s)^{m}+s^{m}(1-s)^{\ell}\right]\right) h(s t) h((1-s) t) \mathrm{d} s \\
= & \int_{0}^{1 / 2}\left[\frac{s^{i}(1-s)^{j}+s^{j}(1-s)^{i}}{s^{\ell}(1-s)^{m}+s^{m}(1-s)^{\ell}}-\frac{i ! j !}{\ell ! m !}\right] \\
& \times\left[s^{\ell}(1-s)^{m}+s^{m}(1-s)^{\ell}\right] h(s t) h((1-s) t) \mathrm{d} s
\end{aligned}
$$

for $t \in(0, \infty)$ and $(i, j) \succ(\ell, m)$. By Lemma 2.3. Lemma 2.4. and the sharp inequality (3.2), we arrive at that the function

$$
\frac{s^{i}(1-s)^{j}+s^{j}(1-s)^{i}}{s^{\ell}(1-s)^{m}+s^{m}(1-s)^{\ell}}-\frac{i ! j !}{\ell ! m !}
$$

is decreasing in $s \in\left(0, \frac{1}{2}\right)$ and has a unique zero $s_{0} \in\left(0, \frac{1}{2}\right)$ for $(i, j) \succ(\ell, m)$. As a result, utilizing Lemma 2.1, we have

$$
\begin{aligned}
S_{i, j ; \ell, m}(t)= & \left(\int_{0}^{s_{0}}+\int_{s_{0}}^{1 / 2}\right)\left[\frac{s^{i}(1-s)^{j}+s^{j}(1-s)^{i}}{s^{\ell}(1-s)^{m}+s^{m}(1-s)^{\ell}}-\frac{i ! j !}{\ell ! m !}\right] \\
& \times\left[s^{\ell}(1-s)^{m}+s^{m}(1-s)^{\ell}\right] h(s t) h((1-s) t) \mathrm{d} s \\
< & h\left(s_{0} t\right) h\left(\left(1-s_{0}\right) t\right) \int_{0}^{1 / 2}\left[\frac{s^{i}(1-s)^{j}+s^{j}(1-s)^{i}}{s^{\ell}(1-s)^{m}+s^{m}(1-s)^{\ell}}-\frac{i ! j !}{\ell ! m !}\right] \\
& \times\left[s^{\ell}(1-s)^{m}+s^{m}(1-s)^{\ell}\right] \mathrm{d} s \\
= & h\left(s_{0} t\right) h\left(\left(1-s_{0}\right) t\right) \int_{0}^{1 / 2}\left(s^{i}(1-s)^{j}+s^{j}(1-s)^{i}\right. \\
& \left.-\frac{i ! j !}{\ell ! m !}\left[s^{\ell}(1-s)^{m}+s^{m}(1-s)^{\ell}\right]\right) \mathrm{d} s \\
= & h\left(s_{0} t\right) h\left(\left(1-s_{0}\right) t\right)\left(\int_{0}^{1 / 2}\left[s^{i}(1-s)^{j}+s^{j}(1-s)^{i}\right] \mathrm{d} s\right. \\
& \left.-\frac{i ! j !}{\ell ! m !} \int_{0}^{1 / 2}\left[s^{\ell}(1-s)^{m}+s^{m}(1-s)^{\ell}\right] \mathrm{d} s\right) \\
= & h\left(s_{0} t\right) h\left(\left(1-s_{0}\right) t\right)\left[B(i+1, j+1)-\frac{i ! j !}{\ell ! m !} B(\ell+1, m+1)\right] \\
= & h\left(s_{0} t\right) h\left(\left(1-s_{0}\right) t\right)\left[\frac{i ! j !}{(i+j+1) !}-\frac{i ! j !}{\ell ! m !} \frac{\ell ! m !}{(\ell+m+1) !}\right] \\
= & 0 .
\end{aligned}
$$

Accordingly, the inequality

$$
\frac{\int_{0}^{1 / 2}\left[s^{i}(1-s)^{j}+s^{j}(1-s)^{i}\right] h(s t) h((1-s) t) \mathrm{d} s}{\int_{0}^{1 / 2}\left[s^{\ell}(1-s)^{m}+s^{m}(1-s)^{\ell}\right] h(s t) h((1-s) t) \mathrm{d} s}<\frac{i ! j !}{\ell ! m !}
$$


is valid and sharp for $t \in(0, \infty)$ and $(i, j) \succ(\ell, m)$. Therefore, by Lemma 2.5 for $(i, j) \succ(\ell, m)$, if and only if $\Omega_{i, j ; \ell, m} \geq \frac{i ! j !}{\ell ! m !}$, the function $-\mathfrak{Y}_{i, j ; \ell, m ; \Omega_{i, j ; \ell, m}}(x)$ is completely monotonic on $(0, \infty)$.

The double inequality (3.1) follows from complete monotonicity of the functions $\pm \mathfrak{Y}_{i, j ; \ell, m ; \Omega_{i, j ; \ell, m}}(x)$ on $(0, \infty)$. The sharpness of the right hand side inequality in (3.1) follows from the limits

$$
(-1)^{k} x^{k+1} \Phi^{(k)}(x) \rightarrow \begin{cases}k !, & x \rightarrow 0^{+} \\ \frac{k !}{2}, & x \rightarrow \infty\end{cases}
$$

in [12, Lemma 2.2], where $k \geq 0$. The proof of Theorem 3.1 is complete.

\section{Logarithmic CONVEXity of the SEQUenCe of POLygamma FunCtions}

In this section, we present that the sequence of polygamma functions $\psi^{(n)}(x)$ for $n \in \mathbb{N}$ is logarithmic convex.

Theorem 4.1. For fixed $s \in(0, \infty)$ and a variable $t \in(0, \infty)$, the function

$$
\boldsymbol{\Phi}(s, t)=\int_{0}^{\infty} \frac{u^{t}}{1-e^{-u}} e^{-s u} \mathrm{~d} u
$$

is logarithmically convex in $t \in(0, \infty)$. Consequently, the sequence $(-1)^{n+1} \psi^{(n)}(x)$ is logarithmically convex in $n \in \mathbb{N}$ and

$$
\psi^{(m-k)}(x) \psi^{(m+k)}(x) \geq\left[\psi^{(m)}(x)\right]^{2}
$$

for $m>k>0$.

Proof. Two functions $f(u)$ and $g(u)$ are called synchronous on an interval $I$ if

$$
[f(u)-f(v)][g(u)-g(v)] \geq 0, \quad x, y \in I .
$$

The Čebyšev integral inequality [10, p. 40, Theorem 10] reads that

$$
\int_{a}^{b} p(u) f(u) g(u) \mathrm{d} x \int_{a}^{b} p(u) \mathrm{d} x \geq \int_{a}^{b} p(u) f(u) \mathrm{d} x \int_{a}^{b} p(u) g(u) \mathrm{d} x,
$$

where $f(u)$ and $g(u)$ are integrable and synchronous functions on $(a, b)$ and $p(u)$ is a positive and integrable function on $(a, b)$. See also the paper [25] and closely related references therein.

Direct calculation yields

$$
\frac{\partial \ln \boldsymbol{\Phi}(s, t)}{\partial t}=\frac{\int_{0}^{\infty} \frac{u^{t} \ln u}{1-e^{-u}} e^{-s u} \mathrm{~d} u}{\int_{0}^{\infty} \frac{u^{t}}{1-e^{-u}} e^{-s u} \mathrm{~d} u}
$$

and

$$
\frac{\partial^{2} \ln \boldsymbol{\Phi}(s, t)}{\partial t^{2}}=\frac{\int_{0}^{\infty} \frac{u^{t}(\ln u)^{2}}{1-e^{-u}} e^{-s u} \mathrm{~d} u \int_{0}^{\infty} \frac{u^{t}}{1-e^{-u}} e^{-s u} \mathrm{~d} u-\left[\int_{0}^{\infty} \frac{u^{t} \ln u}{1-e^{-u}} e^{-s u} \mathrm{~d} u\right]^{2}}{\left[\int_{0}^{\infty} \frac{u^{t}}{1-e^{-u}} e^{-s u} \mathrm{~d} u\right]^{2}} .
$$

Applying $f(u)=g(u)=\ln u$ and $p(u)=\frac{u^{t}}{1-e^{-u}} e^{-s u}$ on the interval $(a, b)=(0, \infty)$ to the inequality (4.1) leads to

$$
\int_{0}^{\infty} \frac{u^{t}(\ln u)^{2}}{1-e^{-u}} e^{-s u} \mathrm{~d} u \int_{0}^{\infty} \frac{u^{t}}{1-e^{-u}} e^{-s u} \mathrm{~d} u \geq\left[\int_{0}^{\infty} \frac{u^{t} \ln u}{1-e^{-u}} e^{-s u} \mathrm{~d} u\right]^{2}
$$


which means $\frac{\partial^{2} \ln \boldsymbol{\Phi}(s, t)}{\partial t^{2}} \geq 0$. Hence, the function $\boldsymbol{\Phi}(s, t)$ is logarithmically convex in $t \in(0, \infty)$. The proof of Theorem 4.1 is complete.

\section{REMARKS}

Finally, we list several remarks.

Remark 5.1. What is the necessary and sufficient condition on $\Omega_{i, j ; \ell, m}$ such that the function $\mathfrak{Y}_{i, j ; \ell, m ; \Omega_{i, j ; \ell, m}}(x)$ defined in 1.1 is completely monotonic on $(0, \infty)$ ?

What is the sharp lower bound of the left hand side inequality in (3.1)?

Remark 5.2. For $\mathbb{N}_{0}=\{0\} \cup \mathbb{N}, n>2$, and two nonnegative integer tuples $\boldsymbol{\alpha}=$ $\left(\alpha_{1}, \alpha_{2}, \ldots, \alpha_{n}\right) \in \mathbb{N}_{0}^{n}$ and $\boldsymbol{\beta}=\left(\beta_{1}, \beta_{2}, \ldots, \beta_{n}\right) \in \mathbb{N}_{0}^{n}$ with $\boldsymbol{\alpha} \succ \boldsymbol{\beta}$, let

$$
P_{\boldsymbol{\alpha}, \boldsymbol{\beta} ; C_{\boldsymbol{\alpha}, \boldsymbol{\beta}}}(x)=\prod_{r=1}^{n}\left[(-1)^{\alpha_{r}} \Phi^{\left(\alpha_{r}\right)}(x)\right]-C_{\boldsymbol{\alpha}, \boldsymbol{\beta}} \prod_{r=1}^{n}\left[(-1)^{\beta_{r}} \Phi^{\left(\beta_{r}\right)}(x)\right]
$$

on $(0, \infty)$. One can discuss necessary and sufficient conditions on $C_{\boldsymbol{\alpha}, \boldsymbol{\beta}} \in \mathbb{R}$ such that the functions $\pm P_{\boldsymbol{\alpha}, \boldsymbol{\beta} ; C_{\boldsymbol{\alpha}, \boldsymbol{\beta}}}(x)$ are respectively completely monotonic on $(0, \infty)$.

Remark 5.3. This paper is the ninth one in a series of articles including [12, 14, 15, 16, 17, 19, 20, 23.

\section{REFERENCES}

[1] M. Abramowitz and I. A. Stegun (Eds), Handbook of Mathematical Functions with Formulas, Graphs, and Mathematical Tables, National Bureau of Standards, Applied Mathematics Series 55, Reprint of the 1972 edition, Dover Publications, Inc., New York, 1992.

[2] H. Alzer and J. Wells, Inequalities for the polygamma functions, SIAM J. Math. Anal. 29 (1998), no. 6, 1459-1466; available online at https://doi.org/10.1137/S0036141097325071

[3] N. Batır, On some properties of digamma and polygamma functions, J. Math. Anal. Appl. 328 (2007), no. 1, 452-465; available online at https://doi.org/10.1016/j.jmaa.2006.05. 065

[4] N. Batır, Some new inequalities for gamma and polygamma functions, J. Inequal. Pure Appl. Math. 6 (2005), no. 4, Art. 103, 9 pages; available online at http://www.emis.de/journals/ JIPAM/article577.html.

[5] C. Berg, E. Massa, and A. P. Peron, A family of entire functions connecting the Bessel function $J_{1}$ and the Lambert $W$ function, Constr. Approx. 52 (2020), in press; available online at https://doi.org/10.1007/s00365-020-09499-x

[6] Y.-M. Chu and X.-H. Zhang, Necessary and sufficient conditions such that extended mean values are Schur-convex or Schur-concave, J. Math. Kyoto Univ. 48 (2008), no. 1, 229-238; available online at https://doi.org/10.1215/kjm/1250280982

[7] P. Gao, Some completely monotonic functions involving the polygamma functions, J. Inequal. Appl. 2019, Paper No. 218, 9 pages; available online at https://doi.org/10.1186/ s13660-019-2172-x

[8] P. Gao, Some monotonicity properties of gamma and q-gamma functions, ISRN Math. Anal. 2011, Art. ID 375715, 15 pages; available online at https://doi.org/10.5402/2011/375715

[9] A. W. Marshall, I. Olkin, and B. C. Arnold, Inequalities: Theory of Majorization and its Applications, 2nd Ed., Springer Verlag, New York/Dordrecht/Heidelberg/London, 2011; available online at http://dx.doi.org/10.1007/978-0-387-68276-1

[10] D. S. Mitrinović, Analytic inequalities, In cooperation with P. M. Vasić, Die Grundlehren der mathematischen Wissenschaften, Band 165, Springer-Verlag, New York-Berlin, 1970.

[11] D. S. Mitrinović, J. E. Pečarić, and A. M. Fink, Classical and New Inequalities in Analysis, Kluwer Academic Publishers, Dordrecht-Boston-London, 1993; available online at https: //doi.org/10.1007/978-94-017-1043-5

[12] F. Qi, Complete monotonicity and monotonicity of two functions defined by two derivatives of a function involving trigamma function, HAL preprint (2020), available online at https: //hal.archives-ouvertes.fr/hal-02993723 
[13] F. Qi, Completely monotonic degree of a function involving trigamma and tetragamma functions, AIMS Math. 5 (2020), no. 4, 3391-3407; available online at https://doi.org/10.3934/ math.2020219

[14] F. Qi, Decreasing monotonicity of two ratios defined by three or four polygamma functions, HAL preprint (2020), available online at https://hal . archives-ouvertes.fr/hal-02998414

[15] F. Qi, Lower bound of sectional curvature of manifold of beta distributions and complete monotonicity of functions involving polygamma functions, MDPI Preprints 2020, 2020110315, 22 pages; available online at https://doi.org/10.20944/preprints202011. 0315.v1

[16] F. Qi, Monotonicity and complete monotonicity of two functions constituted via three derivatives of a function involving trigamma function, OSF Preprints (2020), available online at https://doi.org/10.31219/osf.io/whb2q

[17] F. Qi, Monotonicity of a ratio involving trigamma and tetragamma functions, OSF Preprints (2020), available online at https://doi.org/10.31219/osf.io/5rfb8

[18] F. Qi, Monotonicity results and inequalities for the gamma and incomplete gamma functions, Math. Inequal. Appl. 5 (2002), no. 1, 61-67; available online at http://dx.doi.org/10.7153/ mia-05-08

[19] F. Qi, Necessary and sufficient conditions for two functions defined by two derivatives of a function involving trigamma function to be completely monotonic or monotonic, OSF Preprints (2020), available online at https://doi.org/10.31219/osf.io/6ar4p

[20] F. Qi, Some properties of several functions involving polygamma functions and originating from the sectional curvature of the beta manifold, São Paulo J. Math. Sci. 15 (2021), in press; available online at https://doi.org/10.1007/s40863-020-00193-1

[21] F. Qi and R. P. Agarwal, On complete monotonicity for several classes of functions related to ratios of gamma functions, J. Inequal. Appl. 2019, Paper No. 36, 42 pages; available online at https://doi.org/10.1186/s13660-019-1976-z

[22] F. Qi and B.-N. Guo, From inequalities involving exponential functions and sums to logarithmically complete monotonicity of ratios of gamma functions, J. Math. Anal. Appl. 493 (2021), no. 1, Article 124478, 19 pages; available online at https://doi.org/10.1016/j. jmaa.2020.124478

[23] F. Qi, L.-X. Han, and H.-P. Yin, Monotonicity and complete monotonicity of two functions defined by three derivatives of a function involving trigamma function, HAL preprint (2020), available online at https://hal.archives-ouvertes.fr/hal-02998203

[24] F. Qi and C.-J. Huang, Computing sums in terms of beta, polygamma, and Gauss hypergeometric functions, Rev. R. Acad. Cienc. Exactas Fís. Nat. Ser. A Mat. RACSAM 114 (2020), Article 191, 9 pages; available online at https://doi.org/10.1007/s13398-020-00927-y

[25] F. Qi, G. Rahman, S. M. Hussain, W.-S. Du, and K. S. Nisar, Some inequalities of Cebyšev type for conformable $k$-fractional integral operators, Symmetry 10 (2018), no. 11, Article 614, 8 pages; available online at https://doi.org/10.3390/sym10110614.

[26] F. Qi, X.-T. Shi, M. Mahmoud, and F.-F. Liu, Schur-convexity of the Catalan-Qi function related to the Catalan numbers, Tbilisi Math. J. 9 (2016), no. 2, 141-150; available online at http://dx.doi.org/10.1515/tmj-2016-0026

[27] R. L. Schilling, R. Song, and Z. Vondraček, Bernstein Functions, 2nd ed., de Gruyter Studies in Mathematics 37, Walter de Gruyter, Berlin, Germany, 2012; available online at https: //doi.org/10.1515/9783110269338

[28] H.-N. Shi, Two Schur-convex functions related to Hadamard-type integral inequalities, Publ. Math. Debrecen 78 (2011), no. 2, 393-403; available online at https://doi.org/10.5486/ PMD.2011.4777

[29] N. M. Temme, Special Functions: An Introduction to Classical Functions of Mathematical Physics, A Wiley-Interscience Publication, John Wiley \& Sons, Inc., New York, 1996; available online at http://dx.doi.org/10.1002/9781118032572.

[30] D. V. Widder, The Laplace Transform, Princeton University Press, Princeton, 1946.

[31] Y. Wu, F. Qi, and H.-N. Shi, Schur-harmonic convexity for differences of some special means in two variables, J. Math. Inequal. 8 (2014), no. 2, 321-330; available online at http://dx. doi.org/10.7153/jmi-08-23

[32] Z.-H. Yang, Some properties of the divided difference of psi and polygamma functions, J. Math. Anal. Appl. 455 (2017), no. 1, 761-777; available online at https://doi.org/10. $1016 / j \cdot$ jmaa.2017.05.081 
[33] Z.-H. Yang and J.-F. Tian, Monotonicity and inequalities for the gamma function, J. Inequal. Appl. 2017, Paper No. 317, 15 pages; available online at https://doi.org/10.1186/ s13660-017-1591-9

[34] H.-P. Yin, X.-M. Liu, J.-Y. Wang, and B.-N. Guo, Necessary and sufficient conditions on the Schur convexity of a bivariate mean, AIMS Math. 6 (2021), no. 1, 296-303; available online at https://doi.org/10.3934/math.2021018

[35] H.-P. Yin, H.-N. Shi, and F. Qi, On Schur m-power convexity for ratios of some means, J. Math. Inequal. 9 (2015), no. 1, 145-153; available online at https://doi.org/10.7153/ jmi-09-14.

Email address: qifeng618@gmail.com, qifeng618@hotmail.com, qifeng618@qq.com

$U R L$ : https://qifeng618.wordpress.com, https://orcid.org/0000-0001-6239-2968 